\title{
Métrica usando conceitos de química verde para avaliar o uso de produtos químicos em métodos normatizados nacionais e internacionais para determinação do Número de Acidez Total (NAT)
}

\author{
Green chemistry metric to assess the use of chemicals in national and international standardized \\ methods for determining the Total Acid Number (TAN)
}

Métrica utilizando conceptos de química verde para evaluar el uso de productos químicos en los métodos nacionales e internacionales estandarizados para determinar el número de acidez total (NAT)

Paulo André Prata Decoté

ORCID: https://orcid.org/0000-0002-0141-5043 Universidade Federal do Espírito Santo, Brasil

E-mail: paulodecote@ hotmail.com

Luana Negris Zanelato

ORCID: https://orcid.org/0000-0002-9781-5729 Universidade Federal do Espírito Santo, Brasil

E-mail: luananegris@outlook.com

Amanda Puttin Vidoto

ORCID: https://orcid.org/0000-0002-2526-2774 Universidade Federal do Espírito Santo, Brasil

E-mail: amandaputtinvidoto@ hotmail.com

Maria de Fatima Pereira dos Santos

ORCID: https://orcid.org/0000-0001-6165-003X Universidade Federal do Espírito Santo, Brasil

E-mail: maria.f.santos@ufes.br

Maristela de Araújo Vicente

ORCID: https://orcid.org/0000-0002-2575-2386

Universidade Federal do Espírito Santo, Brasil

E-mail: maristela.vicente@ufes.br

\section{Resumo}

Solventes orgânicos são amplamente utilizados em determinações analíticas. Métodos alternativos de análise, redução do uso de solventes tóxicos, e emprego de solventes verdes são alternativas viáveis para reduzir o impacto ambiental. Além disso, critérios para a seleção adequada de um solvente para um processo pode melhorar muito a sustentabilidade de uma análise. O objetivo da pesquisa foi realizar um estudo comparativo de solventes utilizados na determinação de acidez total em óleo em métodos normatizados nacionais e internacionais. Foram avaliados métodos colorimétrico, potenciométrico e termométrico. Foi estabelecido um índice de toxicidade segundo as informações de perigo descritas em cada ficha de segurança química, para avaliar os métodos normatizados. Foram avaliadas 13 normas: 04 nacionais e 09 internacionais. Os resultados mostraram que os métodos normatizados de maior índice de impacto ambiental e a saúde foram o potenciométrico UOP 565 e termométrico ASTM D8045. Os métodos com menores índices foram os colorimétricos ASTM D1093 e ABNT NBR 16430. Os produtos classificados como perigo à saúde foram identificados em $44 \%$ das normas avaliadas. Concluímos que o estudo dos métodos normatizados internacionais e nacionais evidenciou a necessidade de desenvolver métodos para determinação do Número de Acidez Total visando reduzir o impacto na saúde do operador e ao meio ambiente. O desafio de substituição de solventes em métodos normatizados por produtos verdes deve nortear o desenvolvimento de métodos alternativos e sustentáveis para a análise de acidez em óleo.

Palavras-chave: Número de acidez total (NAT); Métodos normatizados; Índice de toxicidade; Petróleo; Óleo.

\footnotetext{
Abstract

Organic solvents are widely used in analytical determinations. Alternative methods of analysis, reduction use of toxic solvents, and use green solvents are viable alternatives to reduce environmental impact. In addition, criteria for the proper a solvent selection for a process can greatly improve the sustainability of an analysis. The objective of the research was to carry out a comparative study of solvents used in the determination total acidity number (TAN) in oil
} 
in national and international standardized methods. Colorimetric, potentiometric and thermometric methods were evaluated. A toxicity index was established according to the hazard information described in each chemical safety sheet, to assess the standardized methods. 13 standards method were evaluated: 04 national and 09 international. The results showed that the standardized methods with the highest index of environmental impact and health were potentiometric UOP 565 and thermometric ASTM D8045. The methods with the lowest indexes were the colorimetric ASTM D1093 and ABNT NBR 16430. The products classified as a health hazard were identified in 44\% of the evaluated standards. We concluded that the study of international and national standardized methods showed the need to develop methods for determining Total Acidity Number in order to reduce the impact on the operator's health and the environment. The challenge of replacing solvents in standardized methods with green products should guide the development of alternative and sustainable methods for the analysis of acidity in oil.

Keywords: Total acidity number (TAN); Standard methods; Toxicity index; Crude oil; Oil.

\section{Resumen}

Los disolventes orgánicos se utilizan ampliamente en determinaciones analíticas. Debido al impacto ambiental, se buscan métodos alternativos de análisis, reducción del uso de solventes tóxicos o uso de solventes verdes. Además, los criterios para la selección adecuada de un disolvente para un proceso pueden mejorar en gran medida la sostenibilidad de un análisis. El objetivo de la investigación fue realizar un estudio comparativo de los solventes utilizados en la determinación de la acidez total en aceite en métodos estandarizados nacionales e internacionales. Se evaluaron métodos colorimétricos, potenciométricos y termométricos. Se estableció un índice de toxicidad según la información de peligro descrita en cada ficha de seguridad química, para evaluar los métodos estandarizados. Se evaluaron 13 estándares: 04 nacionales y 09 internacionales. Los resultados mostraron que los métodos estandarizados con mayor índice de impacto ambiental y salud fueron el potenciométrico UOP 565 y el termométrico ASTM D8045. Los métodos con índices más bajos fueron el colorimétrico ASTM D1093 y ABNT NBR 16430. Los productos clasificados como de riesgo para la salud se identificaron en el $44 \%$ de las normas evaluadas. Concluimos que el estudio de métodos estandarizados internacionales y nacionales mostró la necesidad de desarrollar métodos para determinar el Número de Acidez Total con el fin de reducir el impacto en la salud del operador y el medio ambiente. El desafío de reemplazar los solventes en métodos estandarizados con productos ecológicos debería guiar el desarrollo de métodos alternativos y sostenibles para el análisis de la acidez en el aceite.

Palabras clave: Número de acidez total (NAT); Métodos estandarizados; Índice de toxicidad; Petróleo; Óleo.

\section{Introdução}

A sustentabilidade de um produto ou processo químico é necessariamente o resultado de uma complexa interação de fatores ambientais, tecnológicos e econômicos e é difícil de prever. Guias são necessários para fornecer meios para selecionar caminhos provavelmente úteis para futuras pesquisas e desenvolvimento (Glavič \& Lukman, 2007; Welton, 2015; Clarke et al., 2018). A seleção do solvente adequado é uma questão chave para uma ampla variedade de produtos químicos e processos (Prat et al., 2015; Driver \& Hunter, 2020). Os solventes sustentáveis são de grande interesse tanto na comunidade científica quanto na indústria química, principalmente devido ao impacto dos solventes na poluição, no uso de energia e nas contribuições para a qualidade do ar e as mudanças climáticas (Clarke et al., 2018). As perdas de solvente representam uma parte importante da poluição orgânica e a remoção de solvente representa um grande consumo de energia do processo (Häckl \& Kunz, 2018).

A química verde tem como objetivo reduzir o uso e a produção de substâncias perigosas em processos químicos, ao mesmo tempo em que reduz o consumo de energia e busca fontes renováveis (Silva et al., 2005). Em um esforço para orientar os pesquisadores em direção a esse objetivo, Anastas e Warner (1998) publicaram os 12 princípios da química verde em 1998. Hoje, a química verde é considerada uma ferramenta para introduzir conceitos sustentáveis em nível fundamental e, portanto, voltada para a invenção de novos produtos, rotas e processos, em vez de melhorar os existentes. Portanto, um dos conceitos de química verde busca desenvolver processos químicos mais eficientes e aperfeiçoar os já existentes, para que estes sejam menos nocivos ao ambiente. É recomendável a escolha de reagentes seguros que minimizem o risco potencial de acidentes.

Vários guias foram descritos na literatura para ajudar os pesquisadores a escolher solventes com baixos impactos ambientais, de segurança e saúde (Byrne et al., 2016; Zhang et al., 2019; Jordan et al., 2020; Musarurwa \& Tavengwa, 2020). Compreender as propriedades do solvente é uma parte necessária do desenvolvimento sustentável, e muitos solventes foram, portanto, classificados por suas características ambientais, de segurança e saúde. Essas classificações são o princípio central 
dos guias de seleção de solventes, a maioria dos quais publicados pela indústria farmacêutica por empresas como GSK, AstraZeneca, Pfizer, e Sanofi, e grupos de especialidades como ACS Green Chemistry Institute Pharmaceutical Roundtable (GCI-PR) e Innovative Medicines Initiative (IMI) - CHEM21 (Alfonsi et al., 2008; Henderson et al., 2011; Prat et al., 2013; Prat et al., 2015; Diorazio et al., 2016). Apesar das informações sobre o desempenho dos solventes não estar disponível para todos os solventes, a estratégia de guias de seleção ou índices para classificação é uma ferramenta que vem sendo usada para produzir resultados imediatos no desenvolvimento sustentável.

Muitos solventes orgânicos são tóxicos, prejudiciais à saúde humana e ao meio ambiente (ABNT NBR 14725-2:2019, 2019; GHS, 2019). Nesse sentido, alternativas para implementar solventes verdes, tais como o uso de biosolventes, substituição de solventes perigosos por aqueles com melhor propriedade, dentre outros, devem ser praticadas (Welton, 2015; Clarke et al., 2018).

Métodos normatizados nacionais e internacionais utilizam solventes e reagentes com diferentes níveis de toxicidade que requerem avaliações para sua substituição. Uma alternativa poderia ser a avaliação comparativa entre métodos normatizados quanto ao desempenho ambiental. Um exemplo é a determinação do Número de Acidez Total (NAT) em amostras de óleo, onde são descritas normas colorimétricas, potenciométrica e termométrica (Speight, 2014a). A norma de titulação potenciométrica ASTM D664 é amplamente utilizada para a determinação do NAT em petróleos, produtos de petróleos e óleos lubrificantes (Oasmaa et al., 2010; Speight, 2014a; Speight, 2014b; Fernandes et al., 2019). Este método, no entanto, possui desvantagens como a produção de grande quantidade de resíduos porque os reagentes não podem ser recuperados e reutilizados, prejudicando o meio ambiente (Parisotto et al., 2010; Baig et al., 2013; Rivera-barrera et al., 2020).

Considerando a relevância na determinação do NAT no controle de processos em diversas matrizes, o objetivo desse estudo foi comparar métodos normatizados nacionais e internacionais aplicados na determinação do NAT quanto à exposição à solventes tóxicos. Foi apresentado um índice de toxicidade dos solventes para contribuir com o aprimoramento desses métodos.

\section{Metodologia}

\subsection{Normas Nacionais e Internacionais}

Foram avaliados os reagentes utilizados em métodos normatizados internacionais e nacionais para determinação da acidez total. Método termométrico: ASTM D8045 (ASTM D8045-17e1, 2018)). Métodos potenciométricos: ASTM D664 (ASTM D664-18e2, 2018); UOP 565 (UOP 565-05, 2005); ABNT NBR 14448 (ABNT NBR 14448:2013, 2013); DIN EN 12634 (DIN EN 12634:1999-02, 1999). Métodos colorimétricos: ASTM D1093 (ASTM D1093-11(2017)); ASTM D974 (ASTM D974-14e2, 2014), UOP 587 (UOP 587-92, 1992); ABNT NBR 14248 (ABNT NBR 14248:2009, 2009); ABNT NBR 14543 (ABNT NBR 14543:2009, 2009); ABNT NBR 16430 (ABNT NBR 16430:2015, 2015); IP 1 (IP 1 - 94(2004), 2004); e DIN ISO 6618 (DIN ISO 6618:2015-07, 2015). As normas ASTM foram adquiridas no site www.periodicos.capes.br e as demais foram adquiridas com recursos de projeto de pesquisa.

Algumas normas apresentam procedimentos para produtos específicos, nomeando internamente como "método de ensaio A" e "B", por exemplo, de acordo com o tipo de amostra. Neste estudo, alguns métodos normatizados foram nomeados seguindo esta classificação: ASTM D664 A (produtos de petróleo) e ASTM D664 B (biodiesel); UOP 565 A (amostra óleo médio e leve) e UOP 565 (amostra óleo pesado); ABNT NBR 14448 A (produtos de petróleo) e ABNT NBR 14448 B (biodiesel); ASTM D1093 A (teste de acidez ) e ASTM D1093 B (teste de basicidade ); ASTM D974 A (teste de acidez), ASTM D974 B (teste de basicidade), ASTM D974 C (número de acidez forte); UOP 587 A (produtos de petróleo), UOP 587 B (amostra livre de mercaptanos e tiofenóis), UOP 587 C (amostras com ácidos naftênicos, incluindo naftenatos de sódio); 
ABNT NBR 14248 A (teste de acidez), ABNT NBR 14248 B (teste de basicidade), ABNT NBR 14248 C (número de acidez forte); IP 1 A (óleos escuros, óleos com alta viscosidade e petrolatos), IP 1 B (óleos claros e com baixa viscosidade); DIN 6618 A (teste de acidez), DIN 6618 B (teste de basicidade), DIN 6618 C (número de acidez forte).

\subsection{Produtos químicos utilizados nos métodos normatizados nacionais e internacionais}

Os reagentes e solventes que são utilizados nos métodos normatizados nacionais e internacionais para determinação de acidez total são: tolueno; xileno; metanol; etanol; isopropanol; acetona; clorofórmio; n-heptano; dimetilsulfóxido; ácido sulfúrico; ácido clorídrico; hidróxido de sódio; hidróxido de potássio; hidróxido de tetrametilamônio; paraformaldeído; ácido benzoico; sulfato de cobre; hidróxido de bário; cloreto de lítio; biftalato de potássio, alaranjado de metila; fenolftaleina; timolftaleína; azul alcalino 6B; e p-naftolbenzeína. Na Tabela 1 são listados os principais produtos químicos e os métodos normatizados por colorimetria e na Tabela 2 são listados os métodos normativos oficiais potenciométricos e termométrico para determinação de acidez total. 
Tabela 1. Principais produtos químicos usados nos métodos normativos oficiais colorimétricos para determinação de acidez total.

\begin{tabular}{|c|c|c|c|c|c|c|c|c|}
\hline \multirow[t]{2}{*}{ Norma } & \multicolumn{8}{|c|}{ Produtos químicos } \\
\hline & Tolueno & Isopropanol & Metanol & $\mathrm{Ba}(\mathrm{OH})_{2}$ & $\mathrm{CuSO}_{4}$ & $\mathrm{KOH}$ & Ácido Clorídrico & Indicador \\
\hline ASTM D1093 A & - & - & - & - & - & - & - & $\mathrm{a}$ \\
\hline ASTM D1093 B & - & - & - & - & - & - & - & $\mathrm{b}$ \\
\hline UOP 587 A & - & $X$ & $\mathrm{X}$ & - & - & $X$ & - & c \\
\hline UOP 587 B & - & $X$ & $X$ & - & $X$ & $X$ & - & c \\
\hline UOP $587 \mathrm{C}$ & - & $\mathrm{X}$ & $\mathrm{X}$ & - & $\mathrm{X}$ & $X$ & - & $\mathrm{c}$ \\
\hline ABNT NBR 16430 & - & $X$ & - & - & - & - & - & $\mathrm{b}$ \\
\hline IP 1 A & $X$ & $X$ & - & - & - & $\mathrm{X}$ & - & $\mathrm{d}$ \\
\hline IP 1 B & - & $\mathrm{X}$ & - & - & - & $X$ & - & $\mathrm{b}$ \\
\hline ABNT NBR 14248 & $\mathrm{X}$ & $X$ & - & $X$ & - & - & - & $\mathrm{e}$ \\
\hline ABNT NBR 14248 B & $X$ & $X$ & - & - & - & - & $X$ & $\mathrm{e}$ \\
\hline ABNT NBR $14248 \mathrm{C}$ & - & $X$ & - & $X$ & - & - & - & $\mathrm{a}$ \\
\hline ABNT NBR 14543 & $X$ & $X$ & - & $X$ & - & - & - & $\mathrm{e}$ \\
\hline ASTM D974 A & $\mathrm{X}$ & $X$ & - & $\mathrm{X}$ & - & - & - & $\mathrm{e}$ \\
\hline ASTM D974 B & $\mathrm{X}$ & $\mathrm{X}$ & - & - & - & - & $\mathrm{X}$ & e \\
\hline ASTM D974 C & - & $\mathrm{X}$ & - & $\mathrm{X}$ & - & - & - & $\mathrm{a}$ \\
\hline DIN ISO 6618 A & $X$ & $X$ & - & $X$ & - & - & - & $\mathrm{e}$ \\
\hline DIN ISO 6618 B & $\mathrm{X}$ & $X$ & - & - & - & - & $X$ & $\mathrm{e}$ \\
\hline DIN ISO $6618 \mathrm{C}$ & - & $\mathrm{X}$ & - & $\mathrm{X}$ & - & - & - & $\mathrm{a}$ \\
\hline
\end{tabular}

Indicadores: (a): Alaranjado de metila; (b): Fenolftaleína; (c): Timolftaleína; (d): Azul alcalino 6B; (e) p-naftolbenzeína. (X) presença e (-) ausência. Fonte: Autores. 
Tabela 2. Principais produtos químicos usados nos métodos normativos oficiais potenciométricos e termométrico para determinação de acidez total.

\begin{tabular}{|c|c|c|c|c|c|c|c|c|}
\hline \multirow[t]{2}{*}{ Norma } & \multicolumn{8}{|c|}{ Produtos químicos } \\
\hline & Tolueno & Xileno & Isopropanol & Clorofórmio & n-Heptano & $\begin{array}{c}\text { Hidróxido de } \\
\text { tetrametilamônio }\end{array}$ & Ácido Clorídrico & Indicador \\
\hline ASTM D8045* & $\mathrm{X}$ & $\mathrm{X}$ & $\mathrm{X}$ & - & $\mathrm{X}$ & - & - & - \\
\hline ASTM D664 A & $\mathrm{X}$ & - & $\mathrm{X}$ & - & - & - & $\mathrm{X}$ & - \\
\hline ASTM D664 B & - & - & $\mathrm{X}$ & - & - & - & - & - \\
\hline ABNT NBR 14448 A & $\mathrm{X}$ & - & $\mathrm{X}$ & - & - & - & $\mathrm{X}$ & - \\
\hline ABNT NBR 14448 B & $\mathrm{X}$ & - & $\mathrm{X}$ & - & - & - & - & - \\
\hline UOP 565 A & $\mathrm{X}$ & - & $X$ & - & - & - & - & - \\
\hline UOP 565 B & $\mathrm{X}$ & - & $X$ & $\mathrm{X}$ & - & - & - & - \\
\hline DIN EN 12634 & $\mathrm{X}$ & - & $\mathrm{X}$ & - & - & $\mathrm{X}$ & - & - \\
\hline
\end{tabular}

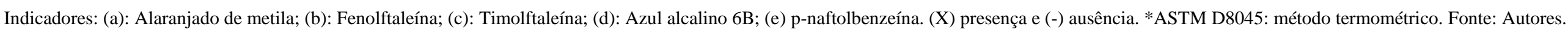




\section{3 Índice de impacto ambiental e de saúde para os solventes e reagentes utilizados nas normas}

\subsubsection{Coleta de informações de perigo}

$\mathrm{O}$ uso de solventes na indústria química está relacionado a uma série de perigos inerentes às suas propriedades: inflamáveis e explosivos, podem ser tóxicos e persistentes no meio ambiente. O índice de impacto ambiental e de saúde (IAS) foi desenvolvido para avaliar os perigos das substâncias químicas usadas nos métodos normatizados durante as determinações de acidez. O índice IAS depende fortemente da disponibilidade de dados sobre propriedades físicas e químicas, toxicidade, aspectos ambientais e de segurança das substâncias a serem avaliadas. Utilizando uma ferramenta de software (Microsoft Excel) as informações das propriedades dos solventes e reagentes foram coletadas no banco de dados da PubChem (https://pubchem.ncbi.nlm.nih.gov/). Foram levadas em consideração as informações: inflamável, corrosivo, irritante, tóxico, perigo a saúde, e perigo ao meio ambiente. Também foi considerado as declarações de perigo quanto ao tipo: física (H225 e H226); saúde (H300, H301, H302, H303, H304, H310, H311, H312, H313, H314, H315, H316, H317, H318, H319, H320, H330, H331, H332, H333, H334, H335, H336, H340, H341, H350, H351, H360, H361, H362, H370, H371, H372 e H373), e de meio ambiente (H400, H401, H402, H410, H411, H412, H413 e H420) (ABNT NBR 14725-2:2019, 2019; GHS, 2019).

\subsubsection{Construção das planilhas de dados}

Uma planilha foi desenvolvida com as informações de segurança e perigo descritas para cada reagente: inflamável; oxidante; corrosivo; irritante; tóxico; perigo para saúde; perigo ambiental (ABNT NBR 14725-2:2019, 2019; GHS, 2019). Para classificação de cada produto químico, foram atribuídos o valor arbitrário " 0 " para ausência da variável. Foi atribuído o valor arbitrário "1” para "Inflamável", "Corrosivo", "Irritante", "Tóxico", "Perigo a saúde", "Perigo ao meio ambiente", H303, H304, H313, H317, H320, H333 e H336; valor arbitrário "2" para H226, H302, H312, H316, H319, H332, H335 e H373; valor arbitrário “3” para H225, H301, H311, H315, H318, H331, H334, H362, H372, H413; valor arbitrário “4” para H314, H341, H351, H361, H371, H401, H402, H411, H412; e valor arbitrário "5" para H300, H310, H330, H340, H350, H360, H370, H400, H410 e H420. A Figura 1 apresenta o fluxograma utilizado para a classificação dos produtos químicos. 
Research, Society and Development, v. 10, n. 5, e25510514894, 2021

(CC BY 4.0) | ISSN 2525-3409 | DOI: http://dx.doi.org/10.33448/rsd-v10i5.14894

Figura 1. Fluxograma de tomada de decisão para classificar os produtos químicos (solventes/reagentes).

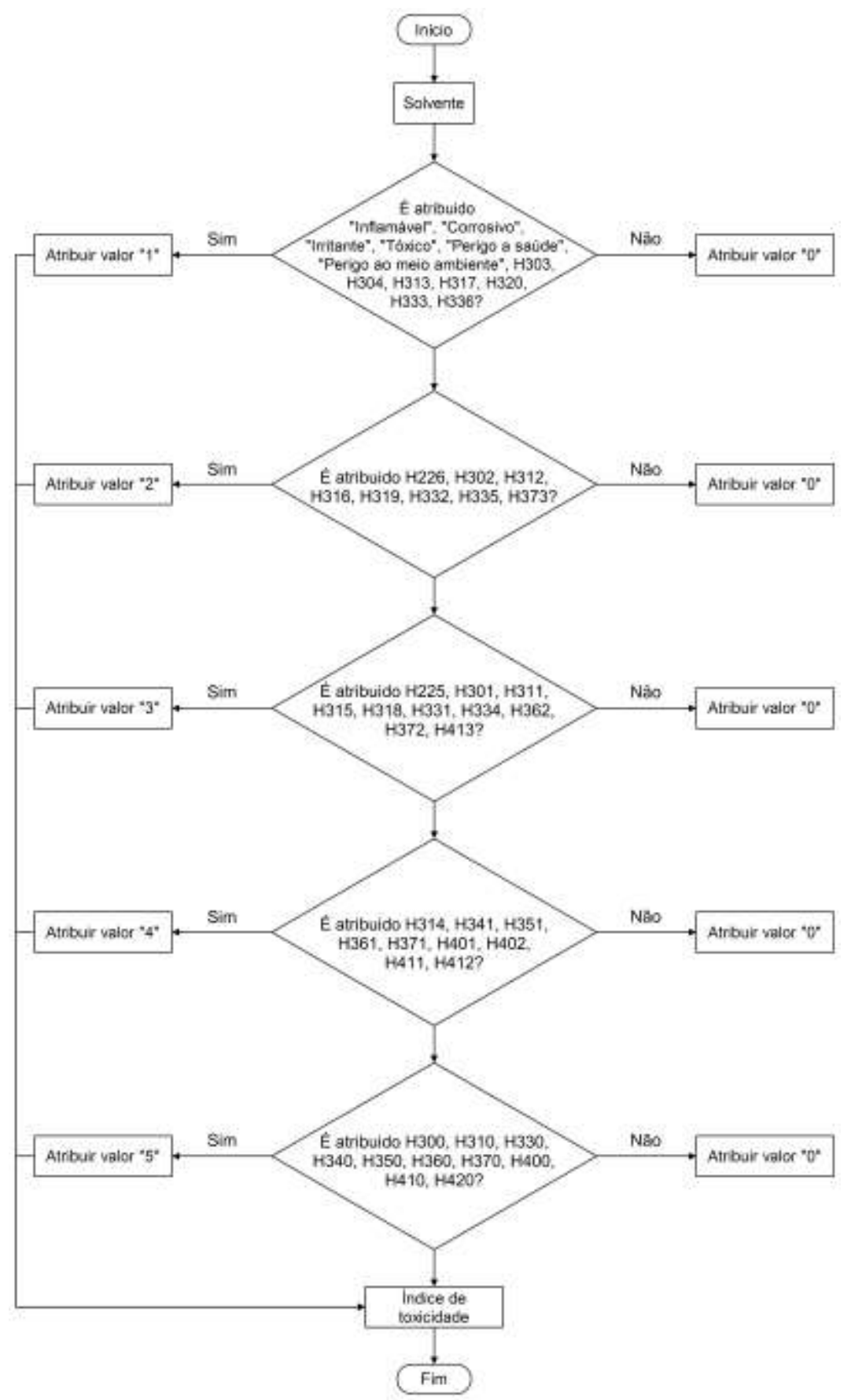

Fonte: Autores.

Após atribuir os valores arbitrário para cada produto químico (solvente, reagente, indicador), fez-se uma somatória dos valores atribuídos a cada produto químico e estabelecido uma porcentagem para comparação, conforme equação 1 , onde $\mathrm{X}$ é o valor arbitrário atribuído para cada variável e n o número de variáveis.

$$
\sum x \cdot \frac{100}{n}
$$


Uma segunda planilha foi construída a partir do levantamento dos produtos químicos utilizados nos métodos normatizados nacionais e internacionais para determinação de acidez em óleo. $\mathrm{O}$ valor obtido na planilha 01 para cada produto químico foi atribuído, e fez-se uma somatória destes valores segundo a equação 1. Foi incluído um método normatizado controle que recebeu valores máximos para cada variável. Este conjunto de dados foi padronizado (média dividido pelo desvio padrão). Para cálculo do Índice de toxicidade, foi adotado a equação 2, onde $\mathrm{X}_{\text {normatizado }}$ é o valor padronizado de cada método normatizado e $\mathrm{X}_{\text {controle }} \mathrm{O}$ valor padronizado da variável método normatizado controle.

$$
\text { Indice de toxicidade }=\frac{X_{\text {normatizado }}}{X_{\text {controle }}} \cdot 100
$$

\section{Resultados e Discussão}

\subsection{Desenvolvimento do índice de impacto para avaliação dos métodos normativos}

Para a classificação dos produtos químicos foi desenvolvido um índice de impacto levando em consideração aspectos como o impacto ambiental, inflamabilidade, e impacto a saúde humana (Henderson et al., 2011). Por meio desse processo, foi utilizado as frases de perigo do Sistema Globalmente Harmonizado (ABNT NBR 14725-2:2019, 2019; GHS, 2019). Os valores atribuídos para cada variável levaram em consideração o alto risco de problemas de saúde humana, recebendo valores maiores aqueles que apresentam riscos relacionados à carcinogenicidade, mutagenicidade ou toxicidade para a reprodução. $\mathrm{O}$ maior valor de classificação foi atribuído para o risco ambiental porque foi considerado relevante no quesito sustentabilidade química.

O conjunto de produtos químicos foi composto por reagentes, solventes e indicadores, que são necessários para a execução do método normativo. O índice não se restringiu apenas a solventes orgânicos, uma vez que para estabelecer um índice comparativo, é importante levar em consideração todos os componentes do processo.

Os indicadores químicos são utilizados nos processos industriais e em métodos colorimétricos. Os indicadores são corantes, e alguns são recalcitrantes, difíceis de biodegradar e são tóxicos para espécies aquáticas, animais e seres humanos. Devido a sua alta solubilidade em água, eles causam contaminação nos sistemas aquáticos locais quando descartados sem tratamento (Hassaan et al., 2017; Bayomie et al., 2020). Portanto, os indicadores utilizados nos métodos normatizados foram incluídos na classificação.

Apesar dos índices de impacto ambiental utilizados por vários autores levar em consideração apenas os solventes orgânicos, nesse trabalho levou-se em consideração também os produtos químicos inorgânicos, visando uma avaliação mais robusta dos impactos ao meio ambiente e à saúde (Byrne et al., 2016; Zhang et al., 2019; Jordan et al., 2020; Musarurwa \& Tavengwa, 2020). Apesar de serem utilizadas, em alguns casos, quantidades relativamente baixas desses produtos, o risco da exposição existe e por isto foi considerado na classificação.

No conjunto de 25 produtos químicos analisados (5 indicadores e 20 reagentes orgânicos e inorgânicos), nenhum dos produtos foi classificado como oxidante. Para os demais, a classificação foi: $12,0 \%$ perigo ao meio ambiente; $24,0 \%$ tóxicos; $32,0 \%$ inflamável; 36,0\% corrosivo; 44,0\% perigo à saúde; e 60,0\% irritante, conforme apresentado na Figura 2. Apesar do perigo ao meio ambiente apresentar a menor participação no grupo, esta classificação é bastante relevante pelo potencial de desastre que representa. A próxima etapa foi avaliar a distribuição do perigo em cada classificação utilizando as frases de aviso do sistema GHS. 
Figura 2. Classificação dos 25 produtos químicos utilizados nas 13 normas e seus respectivos métodos para determinação de acidez total em óleo.

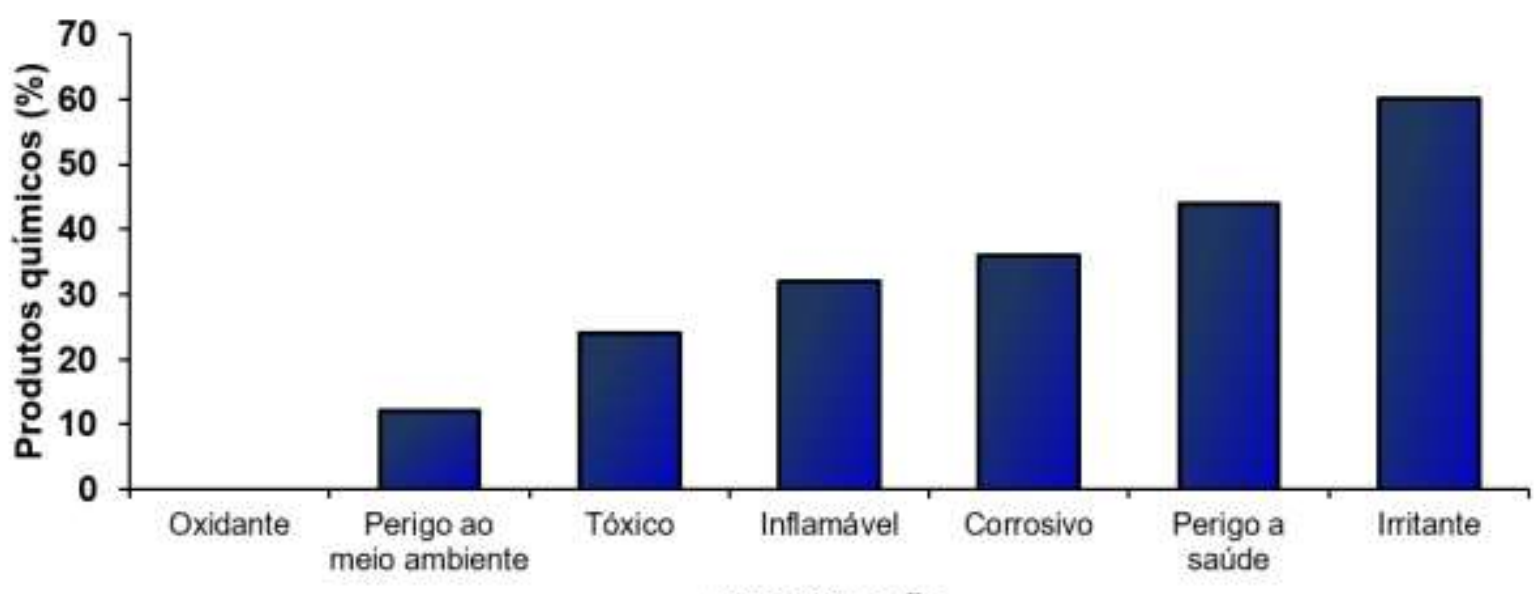

\section{Classificação}

Fonte: Autores.

\subsection{Resultados da classificação utilizando frases de perigo}

As frases de perigo (Hazard statement) identificadas como "H" seguido por 3 dígitos, identificam o tipo de perigo. Para desenvolver a métrica deste estudo, foram atribuídos valores arbitrários segundo a gravidade de cada perigo. Para avaliar a gravidade foram consideradas as categorias de cada frase de perigo, que variaram de 1 (categoria mais grave) até 5 (categoria mais leve). As frases de perigo descrevem principalmente riscos físicos (“H2xx”), riscos à saúde ("H3xx”) e riscos ambientais (“H4xx”). Os valores arbitrários levaram em consideração características como efeitos duradouros, afetar a fertilidade, nocivo por ingestão e inalação, afetar órgãos e toxicidade para organismos aquáticos. Desta forma, foram selecionadas as frases de perigo e atribuído os valores para possibilitar uma classificação numérica.

$\mathrm{Na}$ análise dos produtos químicos, em relação aos riscos à saúde, não foram encontrados representantes dos perigos H303 (Pode ser prejudicial se ingerido), H313 (Pode ser nocivo em contato com a pele), H316 (Causa irritação leve na pele), H320 (causa irritação nos olhos), H330 (fatal se inalado), H333 (pode ser prejudicial se inalado), H334 (Pode causar sintomas de alergia ou asma ou dificuldades respiratórias se inalado), H340 (Pode causar defeitos genéticos), H362 (pode causar danos a crianças amamentadas) e H371 (Pode causar danos aos órgãos). Dessas frases de perigo, 03 frases são de categoria 1 (H330, H334 e H340), 02 frases de categoria 2 (H320 e H371), 02 frases de categoria 3 (H316 e H362) e 03 frases de categoria 5 (H303, H313 e H333). Os resultados mostram que H315 (Corrosão / irritação da pele), H319 (Lesões oculares graves / irritação), H314 (Corrosão / irritação da pele), H302 (Toxicidade aguda, oral) e H335 (Irritação do trato respiratório, exposição única) representam 40,0\%,36,0\%,28,0\%,20,0\% e 20,0\%, respectivamente, conforme apresentado na Figura 3. As frases de perigo H350 e H351 (carcinogenicidade), H360 e H361 (toxicidade reprodutiva) e H370 (toxicidade para órgãos-alvo específicos - exposição única) estão representadas em 32,0 \% dos produtos analisados. 
Figura 3. Frequência (\%) de frases de risco à saúde no grupo de 25 produtos químicos utilizado nas 13 normas e seus respectivos métodos para determinação de acidez total em óleo.

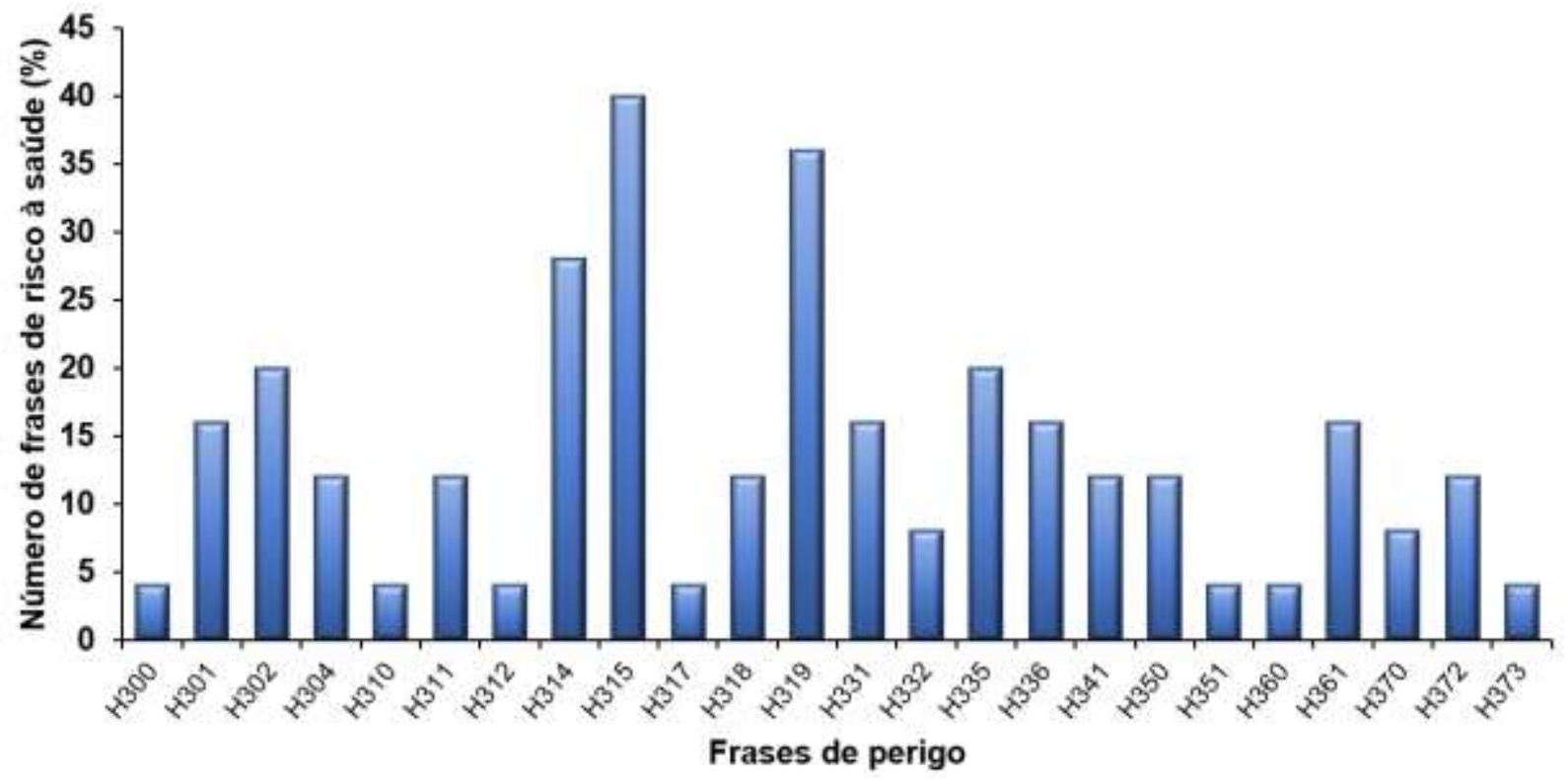

Fonte: Autores.

A atribuição de H351 é atribuído ao clorofórmio, que é um solvente largamente utilizado nos laboratórios para síntese orgânica e outros processos industriais. O uso de solventes tem vantagens e desvantagens, e a substituição em um processo ou determinação leva em consideração aspectos como sustentabilidade, segurança, e viabilidade econômica (Jordan et al., 2020). Atualmente diversas alternativas promissoras de substituição destes solventes estão em estudo como por exemplo, líquidos iônicos, solventes eutéticos profundos (DEP, do inglês deep eutectic solvents), dentre outros (Clarke et al., 2018; Jordan et al., 2020).

No grupo de risco ambiental, estão destacadas as frases H400 (perigoso aquático agudo), H410, H411 e H412 (perigoso aquático crônico). O n-heptano é um exemplo desse grupo de risco ambiental relacionado no grupo de produtos químicos utilizados nos métodos normatizados para determinação de acidez. Byrne et al. (2016) relataram o aumento do uso de n-heptano em substituição a outros solventes, como n-hexano (neurotóxico) e n-pentano (volátil e inflamável). Portanto, o gerenciamento e índices de toxicidade podem orientar usuários ao uso de solventes mais ecológicos e processos menos nocivos ao ambiente e usuários.

\subsection{Estudo comparativo do índice de impacto nos métodos normativos para determinação de acidez em óleo}

Os métodos normatizados para determinação de acidez apresentam diferenças quanto ao tipo e volume de amostra e princípio (termométrico, potenciométrico e colorimétrico). Neste estudo foi avaliado apenas os aspectos de uso de produtos químicos em cada método normatizado para uma indicação de impacto ao meio ambiente e usuário. Uma identificação de perigos (inflamável, oxidante, corrosivo, irritante, tóxico, perigo à saúde, e perigo ao meio ambiente) dos produtos químicos necessários para execução da norma, como destaca a Figura 4, pode indicar ações para mitigar riscos à saúde do operador e no meio ambiente. 
Figura 4. Identificação de perigo em cada método normatizado para determinação do número de acidez total segundo os critérios de inflamável, oxidante, corrosivo, irritante, tóxico, perigo à saúde e perigo ao meio ambiente.

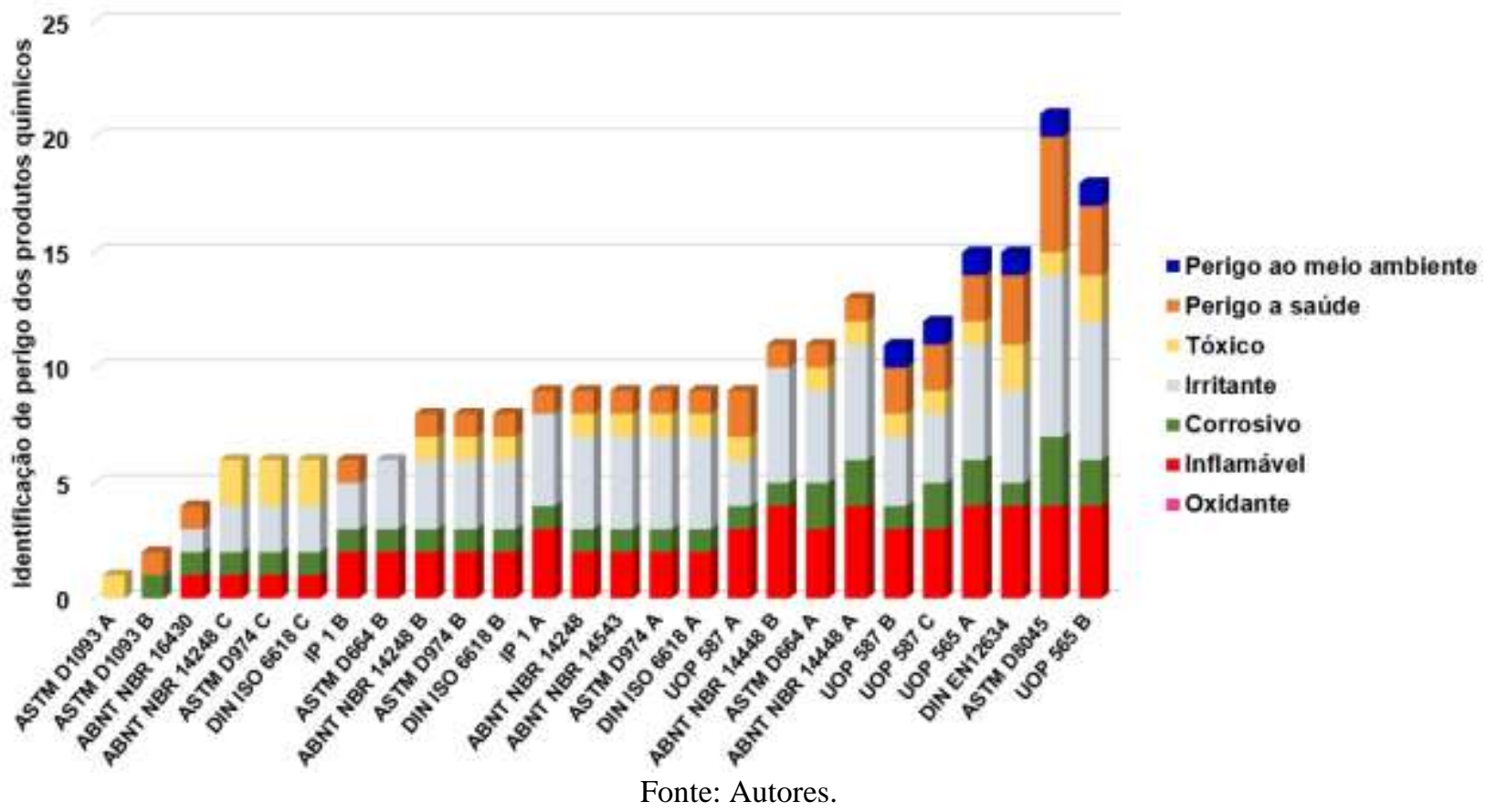

A análise dos produtos químicos utilizados nos métodos normatizados avaliados neste estudo não identificou produtos considerados oxidantes. Com exceção da Norma ASTM D1093 A e B, os demais métodos utilizam produtos inflamáveis. As normas DIN EN 12634, UOP 565 A e B, ABNT NBR 14448 A e B, e ASTM D8045, são as que utilizam o maior número de produtos inflamáveis. As normas UOP 587 B e C, UOP 565 A e B, DIN EN 12634, e ASTM D8045, utilizam produtos considerados perigosos ao meio ambiente. É importante observar que a norma ASTM D664 é amplamente utilizada para determinação de número de acidez total.

Para a classificação de irritante, no grupo de métodos normatizados, apenas a norma ASTM D1093 não utiliza este tipo de produto. A classificação de risco à saúde está presente na maioria dos métodos normatizados, exceção para ASTM D1093 A, ABNT 14248 C, ASTM D974 C, DIN ISO 6618 C e ASTM D664 B. É importante enfatizar que o método ASTM D664 B (biodiesel) não apresentou riscos à saúde principalmente por não fazer uso de tolueno, como o método ASTM D664 A (produtos de petróleo). A norma ASTM D8045 apresentou o maior número de produtos químicos irritantes, corrosivos e perigosos à saúde. Observando estes resultados, uma métrica que represente os produtos químicos que causam maior gravidade à saúde poderá contribuir para destacar os métodos normatizados que necessitam de maior atenção para execução.

Ainda que os métodos normatizados não tratem das questões de segurança ambiental, avaliou-se os produtos químicos utilizados para uma avaliação comparativa de sustentabilidade, impacto à saúde, e ambiental. Para possibilitar o estudo comparativo do impacto ambiental e de saúde dos solventes e reagentes químicos utilizados nos métodos normatizados nacionais e internacionais, foi estabelecido um índice. Este índice foi construído levando em consideração aspectos de sustentabilidade, impacto à saúde, e ambiental.

Além dos critérios analíticos das determinações, uma importante questão é mitigar a exposição do operador aos reagentes e solventes tóxicos. O impacto ambiental é um importante parâmetro de classificação, pois pode ser um indicador na escolha da norma a ser utilizada e privilegiar aquele procedimento experimental mais sustentável e seguro. Considerando que a maioria dos reagentes utilizados nas normas de análise de número de acidez total (NAT) são tóxicos, foi realizado um estudo comparativo para classificar as normas neste parâmetro. A similaridade das normas quanto aos solventes e reagentes usados foi 
avaliada e os resultados estão apresentados na Figura 5.

Figura 5. Índice de impacto ambiental e de saúde das normas nacionais e internacionais para análise de número de acidez total (NAT). Cinza: método termométrico; Azul: método potenciométrico; Verde: método colorimétrico.

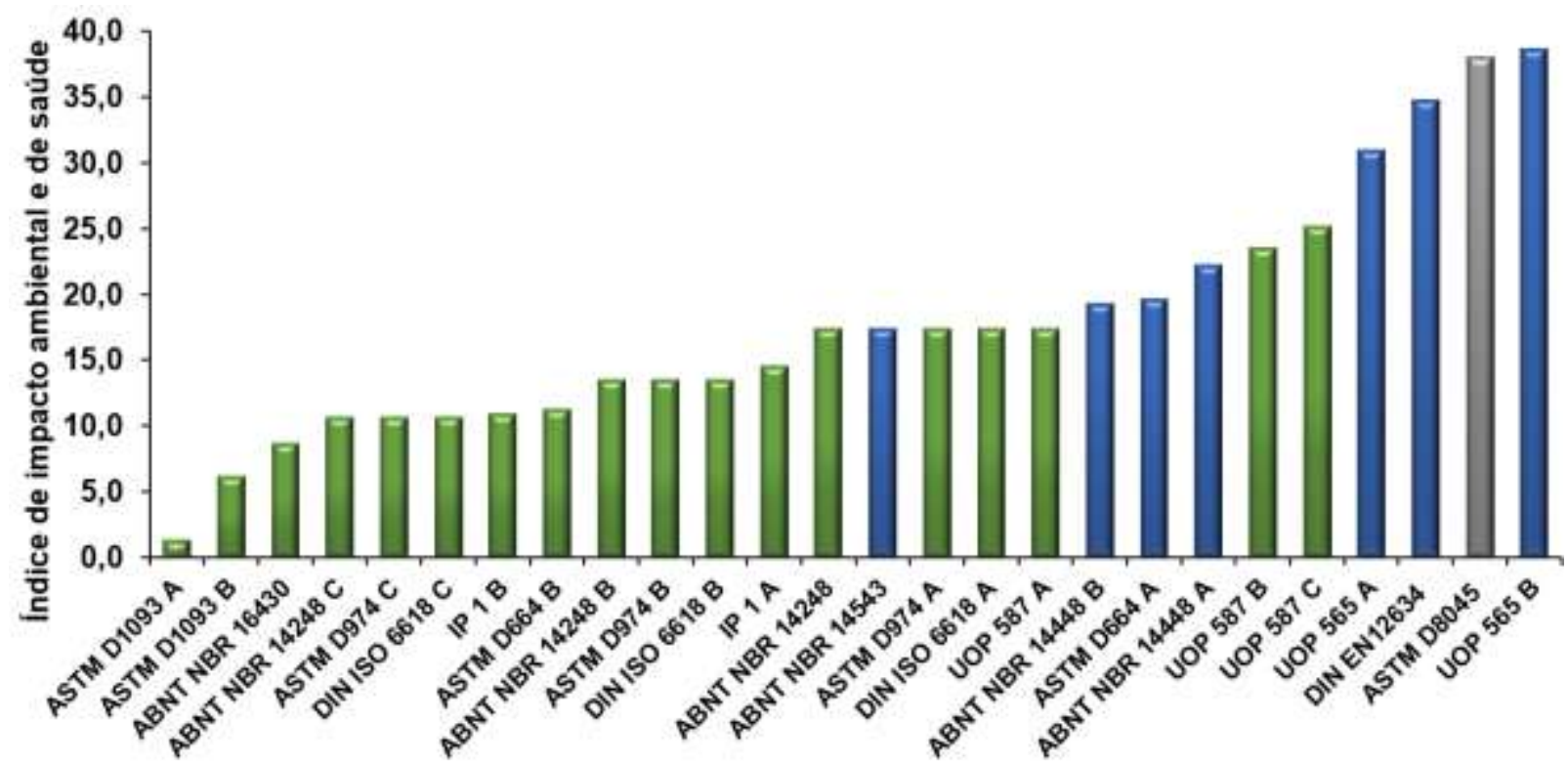

Fonte: Autores.

Os métodos oficiais para determinação do NAT, em razão do seu princípio teórico, podem ser classificados por potenciometria, colorimetria ou termometria (Speight, 2014a). As normas ASTM D664, ABNT NBR 14448, ABNT NBR 14248, ABNT NBR 1093, ASTM D974, UOP 565, UOP 587, DIN ISO 6618 e IP 1 apresentam em seus escopos mais de um procedimento (procedimento A e B ou procedimento A, B e C) a depender do tipo de amostra, e por isto foram considerados separadamente.

A norma com maior índice de impacto ambiental e de saúde foi a norma UOP 565 B (índice de 38,7) seguidas das normas ASTM D8045 (índice de 38,1), DIN EN 12634 (índice de 34,8) e UOP 565 A (índice de 31,0). As normas de maior impacto foram a termométrica (ASTM D8045) e potenciométrica (UOP 565 A e B, DIN EN 12634). A norma colorimétrica de maior índice foi a UOP 587 B e C, com 23,5 e 25,2, respectivamente. Do grupo de normas potenciométrica e colorimétrica, os menores índices foram apresentados pelas normas ABNT NBR 14543 (17,4) e ASTM D1093 A e B $(1,3$ e 6,1), respectivamente.

Se compararmos as Figuras 4 e 5, para as normas UOP 565 B e ASTM D 8045, os resultados aparecem contraditórios quanto ao maior índice de impacto ambiental e número de produtos químicos classificados como perigosos. Esta diferença pode ser explicada pela atribuição de maior valor arbitrário para o desenvolvimento do índice a produtos de maior risco à saúde humana como mutagênicos, carcinogênicos e tóxicos (Alfonsi et al., 2008; Alder et al., 2016). A norma UOP 565 B utiliza quatro produtos nesta categoria (clorofórmio, metanol, tolueno e cloreto de lítio) enquanto a norma ASTM D8045 utiliza três produtos (tolueno, ácido benzoico e paraformaldeído). Se considerarmos a classificação de identificação de perigo, as duas normas têm o mesmo número de produtos inflamáveis (4) e perigo ao meio ambiente (1). Entretanto, a norma ASTM D8045 utiliza 03 produtos corrosivos, 07 irritantes e 05 com perigo à saúde, enquanto a norma UOP 565 B utiliza 02 produtos corrosivos, 06 irritantes e 03 com perigo à saúde. Assim, a análise mais detalhada do tipo de perigo pode contribuir para ações mitigadoras de exposição e nortear o desenvolvimento de métodos alternativos.

Uma das normas mais utilizadas para análise de acidez em óleo, a norma ASTM D664 (índice de 19,7 para o método 
A e 11,3 para o método B) apresentaram valores distantes devido a utilização de tolueno e ácido clorídrico (ASTM D 664 A) na sua execução. Estas normas A e B, também se diferenciam quanto ao escopo, produtos de petróleo e biodiesel, respectivamente. Métodos alternativos tem sido proposto para minimizar o consumo de solventes tóxicos e determinar o valor de acidez total do óleo (Park et al., 2017; Xie et al., 2017; Madsen et al., 2018; Zhang et al., 2019; Rivera-barrera et al., 2020; Macián et al., 2021).

A norma ABNT NBR 14448, embora tenha um procedimento semelhante a norma ASTM D664, teve um índice de impacto maior (índice de 22,3 para o método A e 19,4 para o método B). Essa diferença pode ser explicada pelo procedimento de preparo de amostra (dessalinização) antes da determinação do NAT, que necessita de um grande volume de solventes.

Os métodos colorimétricos são os que apresentaram menor índice de impacto ambiental e de saúde. Em geral, o tipo de amostra abrangido pelos métodos colorimétricos não apresenta dificuldade de solubilização, por isso a quantidade de solventes utilizadas nestes métodos é menor quando comparadas as normas potenciométricas. Apesar desta vantagem, apresenta alguns problemas na execução da técnica em amostras de cor escura, a determinação do ponto final da determinação pode ser um desafio (Aricetti \& Tubino, 2012). Determinações com resultados confiáveis, exatos e precisos, de baixo custo, baixo nível de toxicidade e contaminação ambiental ainda é um desafio para as determinações de número de acidez total.

\section{Conclusão}

Os solventes são um componente intrínseco da maioria dos processos e seus impactos são significativos para a sociedade e o meio ambiente. A busca por solventes considerados menos agressivos e sustentáveis sempre será um desafio. É recomendável que as determinações analíticas desenvolvam processos com solventes mais adequados, ou mitigando os possíveis danos. Índices de impacto podem ajudar na escolha de metodologias que resultem em eficiência e sustentabilidade química.

O índice de impacto apresentado avaliou 13 normas e seus respectivos métodos para determinação de acidez total em óleo. Foi utilizado uma classificação seguindo os paramentos de segurança, saúde e meio ambiente. Os resultados mostraram que as normas UOP 565 (procedimento B), ASTM D8045 e DIN EN 12634 apresentaram alto índice de impacto quando comparados aos demais métodos: 38,7; 38,1; 34,8, respectivamente. Uma das normas mais utilizadas, ASTM D664 também apresentou alto índice de impacto ambiental e à saúde $(19,7)$ entre os métodos potenciométricos. No grupo dos métodos colorimétricos, as normas UOP 587 (procedimento B e C), apresentaram os maiores índices de impacto $(23,5$ e 25,2). A norma ASTM D1093 A, colorimétrica, apresentou menor índice do grupo estudado. Concluímos que a utilização de índices como critério de escolha pode contribuir para busca de determinações robustas e ambientalmente sustentáveis.

\section{Agradecimentos}

O presente trabalho foi realizado com apoio da Coordenação de Aperfeiçoamento de Pessoal de Nível Superior Brasil (CAPES) - Código de Financiamento 001. Os autores agradecem ao Conselho Nacional de Desenvolvimento Científico e Tecnológico (CNPq 313272/2019-0/DT) pelo apoio. Os autores agradecem a Fundação de Amparo à Pesquisa e Inovação do Espírito Santo (FAPES) - (EDITAL FAPES No 26/2018 - Bolsas de Apoio Técnico (AT-NS)). Os autores agradecem à PETROBRAS S.A pelo apoio financeiro para aquisição das normas.

\section{Referências}

ABNT NBR 14248:2009. (2009). Produtos de petróleo - Determinação do número de acidez e basicidade - Método do indicador.

ABNT NBR 14448:2013. (2013). Óleos lubrificantes, produtos de petróleo e biodiesel - Determinação do número de acidez pelo método de titulação 
potenciométrica.

ABNT NBR 14543:2009. (2009). Produtos de petróleo - Determinação do número de acidez por titulação colorimétrica semimicro.

ABNT NBR 14725-2:2019. (2019). Produtos químicos - Informações sobre segurança, saúde e meio ambiente. Parte 2: Sistema de classificação de perigo.

ABNT NBR 16430:2015. (2015). Compostos orgânicos - Determinação da acidez.

Alder, C. M., Hayler, J. D., Henderson, R. K., Redman, A. M., Shukla, L., Shuster, L. E., \& Sneddon, H. F. (2016). Updating and further expanding GSK's solvent sustainability guide. Green Chemistry, 18(13), 3879-3890. https://doi.org/10.1039/C6GC00611F

Alfonsi, K., Colberg, J., Dunn, P. J., Fevig, T., Jennings, S., Johnson, T. A., Kleine, H. P., Knight, C., Nagy, M. A., \& Perry, D. A. (2008). Green chemistry tools to influence a medicinal chemistry and research chemistry based organisation. Green Chemistry, 10(1), 31-36. https://doi.org/10.1039/B711717E

Anastas, P. T., \& Warner, J. C. (1998). Green Chemistry: Theory and Practice. Oxford University Press.

Aricetti, J. A., \& Tubino, M. (2012). A green and simple visual method for the determination of the acid-number of biodiesel. Fuel, 95, 659-661. https://doi.org/10.1016/j.fuel.2011.10.058

ASTM D1093-11(2017). (2017). Standard Test Method for Acidity of Hydrocarbon Liquids and Their Distillation Residues. https://doi.org/10.1520/D1093$11 \mathrm{R} 17$

ASTM D664-18e2. (2018). Standard Test Method for Acid Number of Petroleum Products by Potentiometric Titration. https://doi.org/10.1520/D0664-18E02

ASTM D8045-17e1. (2017). Standard Test Method for Acid Number of Crude Oils and Petroleum Products by Catalytic Thermometric Titration. https://doi.org/10.1520/D8045-17E01

ASTM D974-14e2. (2014). Standard Test Method for Acid and Base Number by Color-Indicator Titration. https://doi.org/10.1520/D0974-14E02

Baig, A., Paszti, M., \& Ng, F. T. T. (2013). A simple and green analytical method for acid number analysis of biodiesel and biodiesel blends based on potentiometric technique. Fuel, 104, 426-432. https://doi.org/10.1016/j.fuel.2012.06.012

Bayomie, O. S., Kandeel, H., Shoeib, T., Yang, H., Youssef, N., \& El-Sayed, M. M. H. (2020). Novel approach for effective removal of methylene blue dye from water using fava bean peel waste. Scientific Reports, 10(1), 1-10. https://doi.org/10.1038/s41598-020-64727-5

Byrne, F. P., Jin, S., Paggiola, G., Petchey, T. H. M., Clark, J. H., Farmer, T. J., Hunt, A. J., McElroy, C. R., \& Sherwood, J. (2016). Tools and techniques for solvent selection: green solvent selection guides. Sustainable Chemical Processes, 4(1), 1-24. https://doi.org/10.1186/s40508-016-0051-z

Clarke, C. J., Tu, W.-C., Levers, O., Brohl, A., \& Hallett, J. P. (2018). Green and sustainable solvents in chemical processes. Chemical Reviews, 118(2), 747800. https://doi.org/10.1021/acs.chemrev.7b00571

DIN EN 12634:1999-02. (1999). Petroleum products and lubricants: Determination of acid number-Non-aqueous potentiometric titration method.

DIN ISO 6618:2015-07. (2015). Petroleum products and lubricants - Determination of acid or base number-Colour-indicator titration method.

Diorazio, L. J., Hose, D. R. J., \& Adlington, N. K. (2016). Toward a more holistic framework for solvent selection. Organic Process Research \& Development, 20(4), 760-773. https://doi.org/10.1021/acs.oprd.6b00015

Driver, M. D., \& Hunter, C. A. (2020). Solvent similarity index. Physical Chemistry Chemical Physics, 22(21), 11967-11975. https://doi.org/10.1039/D0CP01570A

Fernandes, H. A., Freitas, R. R. de, Ribeiro, D. da C., Vicente, M. de A., \& Santos, M. de F. P. dos. (2019). Total acidity in petroleum: A Bibliometric analysis. Research, Society and Development, 8(1), e281505. https://doi.org/10.33448/rsd-v8i1.505

GHS. (2019). Globally Harmonized System of Classification and Labelling of Chemicals (GHS) (Rev.8).

Glavič, P., \& Lukman, R. (2007). Review of sustainability terms and their definitions. Journal of Cleaner Production, 15(18), 1875-1885. https://doi.org/10.1016/j.jclepro.2006.12.006

Häckl, K., \& Kunz, W. (2018). Some aspects of green solvents. Comptes Rendus Chimie, 21(6), 572-580. https://doi.org/10.1016/j.crci.2018.03.010

Hassaan, M. A., El Nemr, A., \& Hassaan, A. (2017). Health and environmental impacts of dyes: mini review. American Journal of Environmental Science and Engineering, 1(3), 64-67. https://doi.org/10.11648/j.ajese.20170103.11

Henderson, R. K., Jiménez-González, C., Constable, D. J. C., Alston, S. R., Inglis, G. G. A., Fisher, G., Sherwood, J., Binks, S. P., \& Curzons, A. D. (2011). Expanding GSK's solvent selection guide-embedding sustainability into solvent selection starting at medicinal chemistry. Green Chemistry, 13(4), 854-862. https://doi.org/10.1039/C0GC00918K

IP 1 - 94(2004). (2004). Determination of acidity, neutralization value - Colour indicator titration method.

Jordan, A., Stoy, P., \& Sneddon, H. F. (2020). Chlorinated Solvents: Their Advantages, Disadvantages, and Alternatives in Organic and Medicinal Chemistry. Chemical Reviews. https://doi.org/10.1021/acs.chemrev.0c00709

Macián, V., Tormos, B., García-Barberá, A., \& Tsolakis, A. (2021). Applying chemometric procedures for correlation the FTIR spectroscopy with the new thermometric evaluation of Total Acid Number and Total Basic Number in engine oils. Chemometrics and Intelligent Laboratory Systems, $208,104215$. https://doi.org/10.1016/j.chemolab.2020.104215 
Research, Society and Development, v. 10, n. 5, e25510514894, 2021

(CC BY 4.0) | ISSN 2525-3409 | DOI: http://dx.doi.org/10.33448/rsd-v10i5.14894

Madsen, R. B., Anastasakis, K., Biller, P., \& Glasius, M. (2018). Rapid determination of water, total acid number, and phenolic content in bio-crude from hydrothermal liquefaction of biomass using FT-IR. Energy \& Fuels, 32(7), 7660-7669. https://doi.org/10.1021/acs.energyfuels.8b01208

Musarurwa, H., \& Tavengwa, N. T. (2020). Emerging green solvents and their applications during pesticide analysis in food and environmental samples. Talanta, 121507. https://doi.org/10.1016/j.talanta.2020.121507

Oasmaa, A., Elliott, D. C., \& Korhonen, J. (2010). Acidity of biomass fast pyrolysis bio-oils. Energy \& Fuels, 24(12), 6548-6554. https://doi.org/10.1021/ef100935r

Parisotto, G., Ferrao, M. F., Müller, A. L. H., Müller, E. I., Santos, M. F. P., Guimaraes, R. C. L., Dias, J. C. M., \& Flores, É. M. M. (2010). Total acid number determination in residues of crude oil distillation using ATR-FTIR and variable selection by chemometric methods. Energy \& Fuels, 24(10), 5474-5478. https://doi.org/10.1021/ef1002974

Park, L. K. E., Liu, J., Yiacoumi, S., Borole, A. P., \& Tsouris, C. (2017). Contribution of acidic components to the total acid number (TAN) of bio-oil. Fuel, 200, 171-181. https://doi.org/10.1016/j.fuel.2017.03.022

Prat, D., Pardigon, O., Flemming, H.-W., Letestu, S., Ducandas, V., Isnard, P., Guntrum, E., Senac, T., Ruisseau, S., \& Cruciani, P. (2013). Sanofi's solvent selection guide: A step toward more sustainable processes. Organic Process Research \& Development, 17(12), 1517-1525. https://doi.org/10.1021/op4002565

Prat, D., Wells, A., Hayler, J., Sneddon, H., McElroy, C. R., Abou-Shehada, S., \& Dunn, P. J. (2015). CHEM21 selection guide of classical-and less classicalsolvents. Green Chemistry, 18(1), 288-296. https://doi.org/10.1039/C5GC01008J

Rivera-barrera, D., Rueda-chacón, H., \& V, D. M. (2020). Prediction of the total acid number (TAN) of colombian crude oils via ATR - FTIR spectroscopy and chemometric methods. Talanta, 206(May 2019), 1-11. https://doi.org/10.1016/j.talanta.2019.120186

Silva, F. M. da, Lacerda, P. S. B. de, \& Jones Junior, J. (2005). Desenvolvimento sustentável e química verde. Química Nova, 28(1), 103-110. https://doi.org/10.1590/S0100-40422005000100019

Speight, J. G. (2014a). High acid crudes. Gulf Professional Publishing.

Speight, J. G. (2014b). The chemistry and technology of petroleum. CRC press.

UOP 565-05. (2005). Acid Number and Naphthenic Acids by Titration (Vol. 05).

UOP 587-92. (1992). Acid Number and Naphthenic Acids by Colorimetric Titration.

Welton, T. (2015). Solvents and sustainable chemistry. Proceedings of the Royal Society A, 471(2183), 20150502. https://doi.org/10.1098/rspa.2015.0502

Xie, W.-Q., Gong, Y.-X., \& Yu, K.-X. (2017). A rapid method for the quantitative analysis of total acid number in biodiesel based on headspace GC technique. Fuel, 210, 236-240. https://doi.org/10.1016/j.fuel.2017.08.070

Zhang, J., Lu, M., Ren, F., Knothe, G., \& Tu, Q. (2019). A greener alternative titration method for measuring acid values of fats, oils, and grease. Journal of the American Oil Chemists' Society, 96(10), 1083-1091. https://doi.org/10.1002/aocs.12281 